PROCEEDINGS OF THE

AMERICAN MATHEMATICAL SOCIETY

Volume 129, Number 2, Pages 609-616

S 0002-9939(00)05788-9

Article electronically published on July 27, 2000

\title{
DIAGONAL TYPE CONDITIONS ON GROUP C*-ALGEBRAS
}

\author{
NICO SPRONK AND PETER WOOD
}

(Communicated by David R. Larson)

\begin{abstract}
Let $G$ be a locally compact group with $\mathbf{C}^{*}(G)$ and $\mathbf{C}_{r}^{*}(G)$ its enveloping and reduced $\mathrm{C}^{*}$-algebras respectively. We show that if $\mathbf{C}^{*}(G)$ is residually finite dimensional, then $G$ is maximally almost periodic, and $\mathbf{C}_{r}^{*}(G)$ is residually finite dimensional if and only if $G$ is both amenable and maximally almost periodic. Letting $\lambda_{G}$ be the left regular representation of $G$, we show that a certain quasidiagonality condition on $\left\{\lambda_{G}(s): s \in G\right\}$ implies that $G$ is amenable.
\end{abstract}

This paper summarizes a few results relating the structures of locally compact groups to diagonal type structures for some of their $\mathrm{C}^{*}$-algebras, namely residual finite dimensionality and quasidiagonality.

The main results in the first section regard such a group $G$ having a residually finite dimensional enveloping $\mathrm{C}^{*}$-algebra, $\mathbf{C}^{*}(G)$. We show that if $\mathbf{C}^{*}(G)$ is residually finite dimensional, then $G$ is maximally almost periodic, and mention a case where the converse does not hold. We obtain a partial converse, though: $G$ is maximally almost periodic exactly when its reduced $\mathrm{C}^{*}$-algebra $\mathbf{C}_{r}^{*}(G)$ is the quotient of a residually finite dimensional $\mathrm{C}^{*}$-algebra which is a quotient of $\mathbf{C}^{*}(G)$. We then obtain the result that $G$ is maximally almost periodic and amenable exactly when $\mathbf{C}_{r}^{*}(G)$ is residually finite dimensional. We conclude the section by exploring the class of groups having residually finite dimensional enveloping $\mathrm{C}^{*}$-algebras. In particular, we examine the stability of this class under free products and amalgams.

The second section records the result that a certain quasidiagonality condition for the unitaries of the left regular representation of $G$ implies that $G$ is amenable. We also extend, to a larger class than discrete groups, Rosenberg's result that if $\mathbf{C}_{r}^{*}(G)$ is quasidiagonal, then $G$ is amenable.

We would like to thank many people who have made comments or suggestions regarding this work. Ken Davidson introduced us to the concept of quasidiagonality. Thierry Giordano brought the useful paper 8 to our attention. Terry Loring informed us of [1]. The first author benefited from conversations with Vladimir Platonov on free products. We would also like to thank our supervisor Brian Forrest for useful conversations about the direction and results of this paper.

Received by the editors April 29, 1999.

1991 Mathematics Subject Classification. Primary 22D05, 22D10, 22D25; Secondary 43A65, 43A07, 46L09.

Key words and phrases. Group $\mathrm{C}^{*}$-algebra, maximal almost periodicity, residual finite dimensionality, amenability, quasidiagonality.

The first author was partially supported by NSERC.

The second author was partially supported by OGS. 


\section{MAXimal ALMOST PERIODICITY AND RESIDUAL FINITE DIMENSIONALiTy}

A locally compact group $G$ is called maximally almost periodic if there exists a separating family of continuous finite dimensional unitary representations of $G$. A $\mathrm{C}^{*}$-algebra $\mathcal{A}$ is called residually finite dimensional if there is a separating family of finite dimensional representations of $\mathcal{A}$. If $\mathcal{A}$ admits a countable separating family of finite dimensional representations, then we could say that $\mathcal{A}$ may be faithfully represented as a block diagonal $\mathrm{C}^{*}$-algebra.

We recall that there is a bijective correspondence between the collection of equivalence classes of continuous unitary representations of a locally compact group $G$ and the collection of equivalence classes of non-degenerate involutive representations of its enveloping $\mathrm{C}^{*}$-algebra $\mathbf{C}^{*}(G)$. If $\sigma: \mathbf{C}^{*}(G) \rightarrow \mathcal{B}(\mathcal{H})$ is an involutive representation on a Hilbert space $\mathcal{H}$, the associated continuous unitary representation of $G$ will be denoted by $\sigma_{G}$; if $\sigma_{G}$ is a continuous unitary representation of $G$, then denote the corresponding representation of $\mathbf{C}^{*}(G)$ by $\sigma$. Note that $\mathbf{C}^{*}(G)$ may be viewed as the completion of $\mathcal{C}_{c}(G)$, the convolution algebra of continuous functions with compact support on $G$, in an appropriate norm. If for $s$ in $G$ and $f$ in $\mathcal{C}_{c}(G)$ we write $s \cdot f(t)=f\left(s^{-1} t\right)$ for $t$ in $G$, then $\sigma(s \cdot f)=\sigma_{G}(s) \sigma(f)$.

Let $\Sigma_{G}$ denote the collection of equivalence classes of continuous unitary representations of $G$. By abuse of notation we will treat subsets of $\Sigma_{G}$ as sets of representatives of elements of $\Sigma_{G}$. Given $\sigma_{G}, \tau_{G}$ in $\Sigma_{G}$, we say that $\sigma_{G}$ is weakly contained in $\tau_{G}$, denoted $\sigma_{G} \prec \tau_{G}$, if $\sigma$ is weakly contained in $\tau$ as a representation of $\mathbf{C}^{*}(G)$, i.e. $\operatorname{ker} \sigma \supset \operatorname{ker} \tau$. If $\sigma_{G} \in \Sigma_{G}$ and $\mathcal{S}$ is a family in $\Sigma_{G}$, write $\sigma_{G} \prec \mathcal{S}$ if $\operatorname{ker} \sigma \supset \bigcap_{\pi_{G} \in \mathcal{S}} \operatorname{ker} \pi$. Let $\widehat{G}$ denote the set of all irreducible representations in $\Sigma_{G}$, and $\widehat{G}_{f}$ denote the set of all finite dimensional representations in $\widehat{G}$. Then $G$ maximally almost periodic if and only if $\widehat{G}_{f}$ is a separating family for $G$.

Theorem 1.1. For a locally compact group $G$, if $\mathbf{C}^{*}(G)$ is residually finite dimensional, then $G$ is maximally almost periodic.

Proof. Let $\mathcal{F}$ be a separating family of finite dimensional non-degenerate involutive representations of $\mathbf{C}^{*}(G)$. Then $\mathcal{F}_{G}$ is separating for $G$. Indeed, if there were $s \in \bigcap_{\pi \in \mathcal{F}} \operatorname{ker} \pi_{G}$, then for all $f$ in $\mathcal{C}_{c}(G)$ we would have $s \cdot f-f \in \bigcap_{\pi \in \mathcal{F}}$ ker $\pi$, a contradiction, unless $s=e$.

Although categorically suggestive, the converse to Theorem 1.1 is false. In fact, it fails for a residually finite discrete group! In [8, p. 137] it is shown that for the residually finite group $G=\mathrm{SL}_{2}(\mathbb{Z}[1 / p]), \mathbf{C}^{*}(G)$ is not residually finite dimensional.

We can answer the question posed in [8, p. 137] of whether for a discrete group $G, \mathbf{C}^{*}(G)$ being residually finite dimensional implies that $G$ is residually finite.

Corollary 1.2. If $G$ is a finitely generated (hence discrete) group and $\mathbf{C}^{*}(G)$ is residually finite dimensional, then $G$ is residually finite. However, there exist countable groups for which $\mathbf{C}^{*}(G)$ is residually finite dimensional but $G$ is not residually finite.

Proof. If $\mathbf{C}^{*}(G)$ is residually finite dimensional, then $G$ is maximally almost periodic. If $\pi \in \widehat{G}_{f}$, then $\pi(G)$ is a finitely generated linear group and so is residually finite by [1, Cor. 1]. This shows that $G$ is residually finite.

If $G$ is a non-trivial divisible group, i.e. $G$ is Abelian and $G^{n}=\left\{s^{n}: s \in G\right\}$ is all of $G$ for $n$ in $\mathbb{N}$, then any quotient of $G$ is also divisible, so can be finite only if 
it is trivial. Hence $G$ cannot be residually finite. However, any divisible group $G$ has that its commutative $\mathrm{C}^{*}$-algebra $\mathbf{C}^{*}(G)$ is residually finite dimensional, since its characters separate points. In particular, the additive group of rationals $\mathbb{Q}$ has a residually finite dimensional enveloping $\mathrm{C}^{*}$-algebra, but is not residually finite.

Much of this proof is contained in [9. Prop. 4].

For a locally compact group $G$, let $\lambda_{G}$ denote the left regular representation of $G$ on $\mathbf{L}^{2}(G)$, given for $s$ in $G$ and $\xi$ in $\mathbf{L}^{2}(G)$ by $\lambda_{G}(s) \xi(t)=\xi\left(s^{-1} t\right)$ for almost all $t$ in $G$. Let $\mathbf{C}_{r}^{*}(G)=\lambda\left(\mathbf{C}^{*}(G)\right)$ denote the reduced $C^{*}$-algebra of $G$.

Theorem 1.3. If $G$ is a locally compact group, then the following are equivalent:

(i) $G$ is maximally almost periodic,

(ii) $\lambda_{G} \prec \widehat{G}_{f}$,

(iii) $\lambda: \mathbf{C}^{*}(G) \rightarrow \mathbf{C}_{r}^{*}(G)$ factors through a residually finite dimensional $C^{*}$ algebra.

Proof. (i) $\Rightarrow$ (ii) This is [4, Example 1.11 (ii)].

(ii) $\Rightarrow$ (iii) Let $\rho_{G}=\bigoplus_{\pi_{G} \in \widehat{G}_{f}} \pi_{G}$ and $\mathbf{C}_{f}^{*}(G)=\rho\left(\mathbf{C}^{*}(G)\right)$. Let $\sigma: \mathbf{C}_{f}^{*}(G) \rightarrow$ $\mathbf{C}_{r}^{*}(G)$ be given by $\sigma(\rho(a))=\lambda(a)$ for $a$ in $\mathbf{C}^{*}(G)$. Since $\lambda_{G} \prec \rho_{G}, \sigma$ is welldefined, and is clearly an involutive representation of $\mathbf{C}_{f}^{*}(G)$. Hence the following diagram commutes:

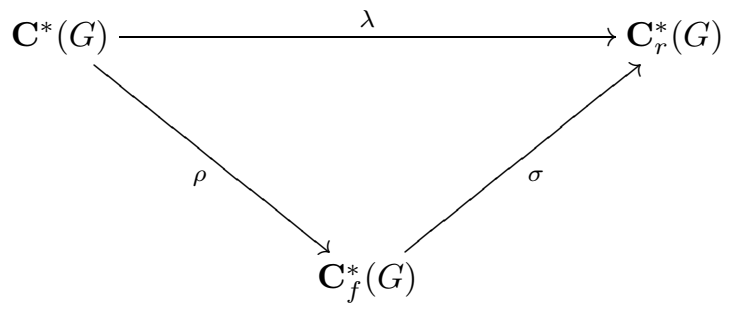

(iii) $\Rightarrow\left(\right.$ i) Let $\rho: \mathbf{C}^{*}(G) \rightarrow \mathcal{B}(\mathcal{H})$ be any representation factoring $\lambda$, where $\rho\left(\mathbf{C}^{*}(G)\right)$ is residually finite dimensional with separating family $\mathcal{F}$ of finite dimensional representations. If $s \in \operatorname{ker} \rho_{G}$, then for all $f \in \mathcal{C}_{c}(G), s \cdot f-f \in \operatorname{ker} \rho \subset \operatorname{ker} \lambda$. Hence $s \cdot f-f=0$ for all $f$ in $\mathcal{C}_{c}(G)$; since $\left.\lambda\right|_{\mathcal{C}_{c}(G)}$ is injective, and thus $s=e$. This means that $\mathcal{F}_{G}$ separates points of $G$ so $G$ is maximally almost periodic.

We can abstractly characterize all locally compact groups $G$ for which $\mathbf{C}_{r}^{*}(G)$ is residually finite dimensional.

Theorem 1.4. A locally compact group $G$ is maximally almost periodic and amenable if and only if $\mathbf{C}_{r}^{*}(G)$ is residually finite dimensional.

Proof. If $G$ is maximally almost periodic and amenable, then it is immediate that $\mathbf{C}^{*}(G) \cong \mathbf{C}_{r}^{*}(G) \cong \mathbf{C}_{f}^{*}(G)$ by [16, Theo. 4.21] and Theorem 1.3 (iii) above, so $\mathbf{C}_{r}^{*}(G)$ is residually finite dimensional.

If $\mathbf{C}_{r}^{*}(G)$ is residually finite dimensional, let $\left\{\pi_{\alpha}\right\}_{\alpha \in A}$ be a separating family of finite dimensional involutive representations of $\mathbf{C}_{r}^{*}(G)$. For $\alpha$ in $A, \pi_{\alpha} \circ \lambda$ is a finite dimensional representation on $\mathbf{C}^{*}(G)$ for which $\left(\pi_{\alpha} \circ \lambda\right)_{G} \prec \lambda_{G}$. Hence $G$ is amenable by [13, Theo. 3]. Then $\mathbf{C}^{*}(G) \cong \mathbf{C}_{r}^{*}(G)$ is residually finite dimensional, so $G$ is maximally almost periodic by Theorem 1.1

For countable groups the above theorem follows from [6, Cor. 4]. 
We have some interest in characterising the class of locally compact groups $G$ for which $\mathbf{C}^{*}(G)$ is residually finite dimensional. We know that all such amenable groups are the maximally almost periodic ones. There are, however, non-amenable groups which are in this class.

If $G$ and $H$ are two discrete groups, denote their free product (or coproduct in the category of groups) by $G \star H$. Provided that at least one of $G$ or $H$ is not a 2 element group and both are non-trivial, it is known that $G \star H$ contains a copy of the free group $F_{2}$, and hence is not amenable.

Proposition 1.5. If $G$ and $H$ are discrete groups such that $\mathbf{C}^{*}(G)$ and $\mathbf{C}^{*}(H)$ are residually finite dimensional, then $\mathbf{C}^{*}(G \star H)$ is residually finite dimensional.

Proof. There is an obvious isomorphism $\mathbf{C}^{*}(G \star H) \cong \mathbf{C}^{*}(G) \star_{\mathbb{C}} \mathbf{C}^{*}(H)$, where $\mathbf{C}^{*}(G) \star_{\mathbb{C}} \mathbf{C}^{*}(H)$ is the unital $\mathbf{C}^{*}$-algebra free product of $\mathbf{C}^{*}(G)$ and $\mathbf{C}^{*}(H)$. This $\mathrm{C}^{*}$-algebra is residually finite dimensional by [11, Theo. 3.2].

We remark that for $F_{2} \cong \mathbb{Z} \star \mathbb{Z}$, the above result is due to Choi [5] (also see 7 . VII.6.1]).

If $G$ and $H$ are groups and there is a group $\Gamma$ which is embedded in each of $G$ and $H$ via homomorphisms $i$ and $j$ respectively, then the amalgam of $G$ and $H$ over $\Gamma$ is $G \star_{\Gamma} H=(G \star H) / N$, where $N$ is the smallest normal subgroup in $G \star H$ containing $\left\{i(a) j(a)^{-1}: a \in \Gamma\right\}$. It is interesting to try to determine if the class of discrete groups having residually finite dimensional $\mathrm{C}^{*}$-algebras is closed under amalgams over common subgroups. We can conclude that this is not the case in general. Note that from $\left[19\right.$, p. 35], $\mathrm{SL}_{2}(\mathbb{Z}) \cong(\mathbb{Z} / 4 \mathbb{Z}) \star_{\mathbb{Z} / 2 \mathbb{Z}}(\mathbb{Z} / 6 \mathbb{Z})$. We do not know if $\mathbf{C}^{*}\left(\mathrm{SL}_{2}(\mathbb{Z})\right)$ is residually finite dimensional. However, by [19, p. 80], for a prime $p$, there is a subgroup $\Gamma(p)$ of $\mathrm{SL}_{2}(\mathbb{Z})$ such that $\mathrm{SL}_{2}(\mathbb{Z}[1 / p]) \cong \mathrm{SL}_{2}(\mathbb{Z}) \star_{\Gamma(p)} \mathrm{SL}_{2}(\mathbb{Z})$. Hence, either $\mathrm{SL}_{2}(\mathbb{Z})$ or $\mathrm{SL}_{2}(\mathbb{Z}[1 / p])$ shows that this class is not closed under amalgams by arbitrary subgroups. We are unaware of whether this class is closed for amalgams over finite subgroups.

If $G$ is a locally compact group for which $\mathbf{C}^{*}(G)$ is residually finite dimensional, then the same is true for any open subgroup $H$ by the fact that $\mathbf{C}^{*}(H)$ may be realized as a subalgebra of $\mathbf{C}^{*}(G)$ by [17] Prop. 1.2]. We are not aware if this property holds for closed subgroups in general.

\section{Quasidiagonality and Amenability}

For our definitions and preliminary results, we follow [14, but suitably generalize them to non-separable Hilbert spaces. A set $\mathcal{S}$ of bounded operators on a Hilbert space $\mathcal{H}$ is called quasidiagonal if for any set $\mathcal{M} \subset \mathcal{H}$ which spans a dense subspace of $\mathcal{H}$, any finite sets $\mathcal{S}_{0} \subset \mathcal{S}, \mathcal{M}_{0} \subset \mathcal{M}$ and $\varepsilon>0$, there is a finite rank projection $p$ on $\mathcal{H}$ such that

$$
\|p a-a p\| \leq \varepsilon \text { for } a \text { in } \mathcal{S}_{0} \text { and }\|p \xi-\xi\|<\varepsilon \text { for } \xi \text { in } \mathcal{M}_{0} .
$$

The following is the non-separable analogue of [14, Lem. 1 (4)].

Proposition 2.1. A set $\mathcal{S}$ of bounded operators on a Hilbert space $\mathcal{H}$ is quasidiagonal if and only if there exists an increasing net of finite rank projections $\left\{p_{\alpha}\right\}_{\alpha \in A}$ tending strongly to the identity operator such that $\lim _{\alpha \in A}\left\|p_{\alpha} a-a p_{\alpha}\right\|=0$ for $a$ in $\mathcal{S}$. 
Proof. If $\mathcal{S}$ is quasidiagonal, let $\mathcal{M}$ be any subset of $\mathcal{H}$ which spans a dense subspace and let $A=\left\{\left(\mathcal{S}_{0}, \mathcal{M}_{0}, \varepsilon\right): \mathcal{S}_{0} \subset \mathcal{S}\right.$ and $\mathcal{M}_{0} \subset \mathcal{M}$ are finite, and $\left.\varepsilon>0\right\}$. There is a natural ordering under which $A$ is a directed set. For $\alpha=\left(\mathcal{S}_{0}, \mathcal{M}_{0}, \varepsilon\right)$ in $A$, let $p_{\alpha}$ be the projection satisfying $(*)$ for the triple $\alpha$. The rest of the proof is straightfoward.

A representation $\rho: \mathcal{A} \rightarrow \mathcal{B}(\mathcal{H})$ of a $\mathrm{C}^{*}$-algebra $\mathcal{A}$ is quasidiagonal if $\rho(\mathcal{A})$ is quasidiagonal. We say that a $\mathrm{C}^{*}$-algebra $\mathcal{A}$ is weakly quasidiagonal if it has a faithful quasidiagonal representation.

It is clear from the above proposition that a residually finite dimensional $\mathrm{C}^{*}$ algebra is weakly quasidiagonal.

We now define two algebras associated with the left regular representation. Let $\mathbf{C}_{\delta}^{*}(G)=\overline{\operatorname{span}}\left\{\lambda_{G}(s): s \in G\right\}$ and $\mathbf{V N}(G)$ be the weak operator closure of $\mathbf{C}_{\delta}^{*}(G)$, or equivalently the weak operator closure of $\mathbf{C}_{r}^{*}(G)$, in $\mathcal{B}\left(\mathbf{L}^{2}(G)\right)$. Note that if $G$ is discrete, then $\mathbf{C}_{\delta}^{*}(G) \cong \mathbf{C}_{r}^{*}(G)$, and if $G$ is Abelian, then $\mathbf{C}_{\delta}^{*}(G) \cong \mathcal{A P}(\widehat{G})$, where $\mathcal{A P}(\widehat{G})$ is the commutative $\mathrm{C}^{*}$-algebra of almost periodic functions on the dual group $\widehat{G}$. It is well known that $\mathbf{V N}(G)$ is the commutant of $\left\{\lambda_{G}^{\prime}(s): s \in G\right\}$, where $\lambda_{G}^{\prime}$ is the right regular representation of $G$ on $\mathbf{L}^{2}(G)$, given for $s$ in $G$ and $\xi$ in $\mathbf{L}^{2}(G)$ by $\lambda_{G}^{\prime}(s) \xi(t)=\Delta(s)^{1 / 2} \xi(t s)$ for almost all $t$ in $G$.

Lemma 2.2. If $G$ is not compact, then $\mathbf{V N}(G)$ contains no non-trivial compact operators.

Proof. Fix $a$ in $\mathbf{V N}(G) \backslash\{0\}$. Find $\xi_{a}$ in $\mathcal{C}_{c}(G)$ such that $\left\|\xi_{a}\right\|_{2}=1$ and $\left\langle a \xi_{a} \mid \xi_{a}\right\rangle \neq$ 0 . (By a numerical range argument we may obtain that $\left|\left\langle a \xi_{a} \mid \xi_{a}\right\rangle\right|>(1-\varepsilon) \frac{1}{2}\|a\|$, for any $\varepsilon>0$.) Let $K=\operatorname{supp}\left(\xi_{a}\right)$. Then we may inductively find a sequence $\left\{t_{n}\right\}_{n \in \mathbb{N}}$ in $G$ such that $K t_{1} \ldots t_{n}$ is disjoint from $K$ and $K t_{1} \ldots t_{k}$ for any $k=$ $1, \ldots, n-1$. Put $\xi_{a, 0}=\xi_{a}$ and for $n$ in $\mathbb{N}, \xi_{a, n}=\lambda_{G}^{\prime}\left(t_{1} \ldots t_{n}\right)^{*} \xi_{a}$. Then $\left\{\xi_{a, n}\right\}_{n \in \mathbb{N} \cup\{0\}}$ is an orthonormal sequence in $\mathbf{L}^{2}(G)$ and

$$
\left\langle a \xi_{a, n} \mid \xi_{a, n}\right\rangle=\left\langle a \lambda_{G}^{\prime}\left(t_{1} \ldots t_{n}\right)^{*} \xi_{a, 0} \mid \lambda_{G}^{\prime}\left(t_{1} \ldots t_{n}\right)^{*} \xi_{a, 0}\right\rangle=\left\langle a \xi_{a, 0} \mid \xi_{a, 0}\right\rangle \neq 0
$$

for all $n$, so $a$ is not compact; for if $a$ were compact, then $\lim _{n \rightarrow \infty}\left\|a \xi_{a, n}\right\|=0$.

The problem of knowing the conditions under which $\mathbf{C}_{r}^{*}(G)$ is (weakly) quasidiagonal has attracted some attention $([14,[18,21], 22])$. It is proved in 18 that for a discrete group $G, \mathbf{C}_{r}^{*}(G)$ is quasidiagonal (as a concrete family of operators) only if $G$ is amenable. We have found that the most natural extension of this result is not an extension to $\mathbf{C}_{r}^{*}(G)$ for locally compact $G$, but to any space of operators containing $\mathbf{C}_{\delta}^{*}(G)$.

Theorem 2.3. If $G$ is a locally compact group for which $\mathbf{C}_{\delta}^{*}(G)$ is weakly quasidiagonal, then $G$ is amenable.

Proof. We may assume that $G$ is not compact. Let $H$ be a countable subgroup of $G$ and $\mathbf{C}_{\lambda_{G}}^{*}(H)=\overline{\operatorname{span}}\left\{\lambda_{G}(s): s \in H\right\}$. Then $\mathbf{C}_{\lambda_{G}}^{*}(H)$ is weakly quasidiagonal. Let $D \subset \mathbf{C}_{\lambda_{G}}^{*}(H)$ be a countable dense subset, and for $d$ in $D \backslash\{0\}$, create $\left\{\xi_{d, n}\right\}_{n \in \mathbb{N} \cup\{0\}}$ as in the lemma above. Then for any separable, $\mathbf{C}_{\lambda_{G}}^{*}(H)$-invariant subspace $\mathcal{H}$ of $\mathbf{L}^{2}(G)$ containing $\left\{\xi_{d, n}: n \in \mathbb{N} \cup\{0\}\right.$ and $\left.d \in D\right\},\left.\mathbf{C}_{\lambda_{G}}^{*}(H)\right|_{\mathcal{H}}$ intersects the compacts on $\mathcal{H}$ trivially. If $\rho$ is a faithful quasidiagonal ${ }^{*}$-representation of $\mathbf{C}_{\lambda_{G}}^{*}(H)$ on a separable Hilbert space $\mathcal{K}$ (i.e. take $\rho$ to be the restriction of some faithful 
quasidiagonal representation to a separable invariant subspace), then so too is $\rho^{(\infty)}$ : $\mathbf{C}_{\lambda_{G}}^{*}(H) \rightarrow \mathcal{B}\left(\mathcal{K}^{(\infty)}\right)$, and

$$
\left.\operatorname{kerid}\right|_{\mathcal{H}}=\left.\operatorname{ker} \pi \circ i d\right|_{\mathcal{H}}=\operatorname{ker} \pi \circ \rho^{(\infty)}=\operatorname{ker} \rho^{(\infty)}
$$

where $\pi$ is the map onto the Calkin algebra. Hence $\left.\mathrm{id}\right|_{\mathcal{H}}$ and $\rho^{(\infty)}$ are approximately unitarily equivalent by Voiculescu's "Weyl-von Neumann" Theorem [20] (see [7. Cor. II.5.6]), so id $\left.\right|_{\mathcal{H}}$ is quasidiagonal by [14, Cor. 3]. Hence $\left.\mathbf{C}_{\lambda_{G}}^{*}(H)\right|_{\mathcal{H}}$ is quasidiagonal as a set of operators on $\mathcal{H}$. Since $\mathcal{H}$ is an arbitrary separable subspace of $\mathbf{L}^{2}(G)$ satisfying the conditions above, it follows that $\mathbf{C}_{\lambda_{G}}^{*}(H)$ is quasidiagonal as a set of operators on $\mathbf{L}^{2}(G)$.

Let $\left\{p_{\alpha}\right\}_{\alpha \in A}$ be a net of finite rank projections on $\mathbf{L}^{2}(G)$ strongly increasing to the identity such that $\lim _{\alpha \in A}\left\|p_{\alpha} a-a p_{\alpha}\right\|=0$ for $a$ in $\mathbf{C}_{\lambda_{G}}^{*}(H)$. Let $\mathcal{U}$ be any ultrafilter on $A$ containing the sets $\left\{\alpha \in A: \alpha \geq \alpha_{0}\right\}$ for each $\alpha_{0}$ in $A$. For any $f$ in $\mathbf{L}^{\infty}(G)$ let $M_{f}$ be the multiplication operator by $f$ on $\mathbf{L}^{2}(G)$. Let $\operatorname{tr}$ be the canonical trace defined on finite rank operators on $\mathbf{L}^{2}(G)$. Then we may define a mean (state) on $\mathbf{L}^{\infty}(G)$ by setting

$$
m(f)=\lim _{\alpha \in U, U \in \mathcal{U}} \frac{1}{\operatorname{tr}\left(p_{\alpha}\right)} \operatorname{tr}\left(p_{\alpha} M_{f} p_{\alpha}\right)
$$

for $f$ in $\mathbf{L}^{\infty}(G)$. We can now follow [22] Prop. 4.2] (see also [7, Prop. VII.7.8]) to see that $m$ is invariant for the left action of $H$ on $\mathbf{L}^{\infty}(G)$. First see that for $t$ in $G$ and $f$ in $\mathbf{L}^{\infty}(G), \lambda_{G}(t) M_{f} \lambda_{G}(t)^{*}=M_{t \cdot f}$. Then for such $f$ and $t$ in $H$ we have

$$
\begin{aligned}
|m(t \cdot f)-m(f)|= & \lim _{\alpha \in U, U \in \mathcal{U}} \frac{1}{\operatorname{tr}\left(p_{\alpha}\right)} \mid \operatorname{tr}\left(p_{\alpha} \lambda_{G}(t) M_{f} \lambda_{G}(t)^{*} p_{\alpha}\right) \\
& -\operatorname{tr}\left(\lambda_{G}(t) p_{\alpha} M_{f} p_{\alpha} \lambda_{G}(t)^{*}\right) \mid \\
= & \lim _{\alpha \in U, U \in \mathcal{U}} \frac{1}{\operatorname{tr}\left(p_{\alpha}\right)} \mid \operatorname{tr}\left(\left[p_{\alpha} \lambda_{G}(t)-\lambda_{G}(t) p_{\alpha}\right] M_{f} \lambda_{G}(t)^{*} p_{\alpha}\right) \\
& \quad+\operatorname{tr}\left(\lambda_{G}(t) p_{\alpha} M_{f}\left[\lambda_{G}(t)^{*} p_{\alpha}-p_{\alpha} \lambda_{G}(t)^{*}\right]\right) \mid \\
\leq & \lim _{\alpha \in U, U \in \mathcal{U}} 2\left\|p_{\alpha} \lambda_{G}(t)-\lambda_{G}(t) p_{\alpha}\right\|\|f\|_{\infty}=0 .
\end{aligned}
$$

For each finite subset $F$ in $G$, let $m_{F}$ be the mean defined above which is invariant for the action of $\langle F\rangle$ on $\mathbf{L}^{\infty}(G)$. Then any weak* cluster point of the net $\left\{m_{F}\right\}_{F \in \mathcal{F}}$, indexed over the collection of all finite subsets of $G$, is a left invariant mean for the action of $G$ on $\mathbf{L}^{\infty}(G)$. Hence $G$ is amenable.

We note that the method used above, of reducing to countable subgroups and applying Voiculescu's theorem, gives a generalization of [18, Theo. A1].

Corollary 2.4. For a discrete group $G$, if $\mathbf{C}_{r}^{*}(G)$ is weakly quasidiagonal, then $G$ is amenable.

For a locally compact group $G$, let $G_{d}$ denote the same group but with the discrete topology. Note that in general $\mathbf{C}_{r}^{*}\left(G_{d}\right)$ is a quotient of $\mathbf{C}_{\delta}^{*}(G)$ by [10] Theo. 2.5] (see also [2, Lem. 2]).

Corollary 2.5. Suppose $G$ is a locally compact group which contains an open subgroup $H$ such that $H_{d}$ is amenable (for example, if $H$ is solvable) and $\mathbf{C}_{\delta}^{*}(G)$ is weakly quasidiagonal. Then $G_{d}$ is amenable. 
Proof. The condition that $G$ has an open subgroup $H$ for which $H_{d}$ is amenable is equivalent to the existence of an isomorphism $\mathbf{C}_{\delta}^{*}(G) \cong \mathbf{C}_{r}^{*}\left(G_{d}\right)$ by [3, Theo. 2]. Then $\mathbf{C}_{r}^{*}\left(G_{d}\right)$ is weakly quasidiagonal so $G_{d}$ is amenable.

We have another extension of Rosenberg's result of a slightly different nature.

Theorem 2.6. If $G$ is a locally compact group with an open normal compact subgroup $K$ and $\mathbf{C}_{r}^{*}(G)$ is weakly quasidiagonal, then $G$ is amenable.

Proof. Let $\mathbf{C}_{r, K}^{*}(G)=\overline{\operatorname{span}}\left\{\lambda\left(\chi_{t K}\right): t \in G\right\}$, where $\chi_{t K}$ is the characteristic function of $t K$. As in [12, p. 217] let $\mathbf{L}_{K}^{2}(G)$ be the closed subspace of elements of $\mathbf{L}^{2}(G)$ which are almost everywhere constant on (left) cosets of $K$, and let $j$ : $\ell^{2}(G / K) \rightarrow \mathbf{L}_{K}^{2}(G)$ be the unitary given by $j \xi=\xi \circ q$, where $q: G \rightarrow G / K$ is the quotient map. Note that we may assume $\mu(K)=1$, where $\mu$ is the Haar measure on $G$, so that $j$ is indeed a unitary. Then the map $a \mapsto j^{*} a j$ is an isomorphism identifying $\mathbf{C}_{r, K}^{*}(G)$ with $\mathbf{C}_{r}^{*}(G / K)$. Since $\mathbf{C}_{r, K}^{*}(G)$ is weakly quasidiagonal, so too is $\mathbf{C}_{r}^{*}(G / K)$. Since $\mathrm{G} / \mathrm{K}$ is discrete, this implies that $G / K$ is amenable; hence so too is $G$, by [16, Prop. 1.13].

We remark that the class of groups having a compact open normal subgroup includes all [IN] groups that have a compact connected component of the identity. (See [15] for a description of [IN] groups.) This class also includes all Lie groups which have a compact connected component of the identity.

\section{ADDED IN PROOF}

A.-T. Lau has kindly pointed out to us that Lemma 2.2 can be found in 23 .

\section{REFERENCES}

[1] R. C. Alperin. An elementary account of Selberg's Lemma. L'Enseignement Math., 33:269273, 1987. MR 89f:20051

[2] E. Bédos. On the $\mathrm{C}^{*}$-algebra generated by the left regular representation of a locally compact group. Proc. Amer. Math. Soc., 120(2):603-608, 1994. MR 94d:22004

[3] M. E. B. Bekka, E. Kaniuth, A. T. Lau, and G. Schlichting. On $\mathrm{C}^{*}$-algebras associated with locally compact groups. Proc. Amer. Math. Soc., 124(10):3151-3158, 1996. MR 96m:22010

[4] M. E. B. Bekka, A. T. Lau, and G. Schlichting. On invariant subalgebras of the FourierStieljes algebra of a locally compact group. Math. Ann., 294:513-522, 1992. MR 93k:43006

[5] M. D. Choi. The full C*-algebra of the free group on two generators. Pacific J. Math., 87:4148, 1980. MR 82b:46069

[6] M. Dadarlat. On the approximation of quasidiagonal $\mathrm{C}^{*}$-algebras. J. Funct. Anal. 167:69-78, 1999. CMP 2000:01

[7] K. R. Davidson. $C^{*}$-algebras by example, volume 6 of Fields Institute Monographs. American Mathematical Society, 1996. MR 97i:46095

[8] P. de la Harpe. Operator algebras, free groups and other groups. Astérisque, 232:121-153, 1995. MR 97m:46092

[9] P. de la Harpe, A.G. Robertson, and A. Valette. On exactness of group C*-algebras. Quart. J. Math. Oxford (2), 45:499-513, 1994. MR 96g:46049

[10] C. F. Dunkl and D. E. Ramirez. C*-algebras generated by Fourier-Stieljes transforms. Trans. Amer. Math. Soc., 164:435-441, 1972. MR 46:9646

[11] R. Exel and T. A. Loring. Finite-dimensional representations of free product $\mathrm{C}^{*}$-algebras. Internat. J. Math., 3(4):469-476, 1992. MR 93f:46091

[12] P. Eymard. L'algèbre de Fourier d'un groupe localement compact. Bull. de la Soc. Math. France, 92:181-236, 1964. MR 37:4208

[13] J. M. G. Fell. Weak containment and Kronecker products of group representations. Pacific J. Math., 13:503-510, 1969. MR 27:5865 
[14] D. Hadwin. Strongly quasidiagonal C*-algebras. J. Operator Theory, 18:3-15, 1987. MR 89d:46060

[15] T. W. Palmer. Classes of nonabelian, noncompact, locally compact groups. Rocky Mountain J. Math., 8(4):683-741, 1978. MR 81j:22003

[16] A. T. Paterson. Amenability, volume 29 of Mathematical Surveys and Monographs. American Mathematical Society, 1988. MR 90e:43001

[17] M. A. Rieffel. Induced representations of $\mathrm{C}^{*}$-algebras. Advan. Math., 13:176-257, 1974. MR 50:5489

[18] J. Rosenberg. Quasidiagonality and nuclearity. J. Operator Theory, 18:15-18, 1987. Appendix to Strongly quasidiagonal $C^{*}$-algebras by D. Hadwin. MR 89d:46060

[19] J.-P. Serre. Trees. Springer, 1980. Translated by J. Stillwell from Arbres, Amalgams, $\mathrm{SL}_{2}$. MR 82c:20083

[20] D. Voiculescu. A non-commutative Weyl-Von Neumann theorem. Rev. Roumaine de Math. Pures Appl., 21:97-113, 1976. MR 54:3427

[21] D. Voiculescu. Around quasidiagonal operators. Integr. Equat. Oper. Th., 17:137-149, 1993. MR 94e:47029

[22] S. Wassermann. Exact $C^{*}$-algebras and related topics, volume 19 of Lecture Notes Series. Seoul National University, Research Institute of Mathematics, Global Analysis Research Center, 1994. MR 95b:46081

[23] C. Chou, A.-T. Lau, and J. Rosenblatt, Approximation of compact operators by sums of translation. Ill. J. Math., 29(2):340-350, 1985. MR 86g:22007

Department of Pure Mathematics, University of Waterloo, Ontario, Canada N2L 3G1

E-mail address: nspronk@math.uwaterloo.ca

Department of Pure Mathematics, University of Waterloo, Ontario, Canada N2L 3G1

E-mail address: pwood@math.uwaterloo.ca 